\title{
Enhancement of the Electromagnetic Wave Shielding Effectiveness by Geometry-Controlled Carbon Coils
}

\author{
Gi-Hwan Kang1, Sung-Hoon Kim², Saehyun Kim² \\ ${ }^{1}$ Center for Green Fusion Technology and Department of Engineering in Energy \& Applied Chemistry, Silla \\ University, Busan 617-736, Republic of Korea \\ ${ }^{2}$ Onnuri International Christian Academy, Yangsan, Kyungnam, 626-813, Republic of Korea \\ Email: shkim@silla.ac.kr
}

Received November 2014

\begin{abstract}
Carbon microcoils were deposited onto $\mathrm{Al}_{2} \mathrm{O}_{3}$ substrates using $\mathrm{C}_{2} \mathrm{H}_{2} / \mathrm{H}_{2}$ as source gases and $\mathrm{SF}_{6}$ as an incorporated additive gas in a thermal chemical vapor deposition system. At as-grown state, the carbon coils (d-CCs) show the diverse geometry. The geometry-controlled carbon microcoils (g-CMCs) could be obtained by manipulating the injection time of $\mathrm{SF}_{6}$ in $\mathrm{C}_{2} \mathrm{H}_{2}$ source gas. The d-CCs with polyurethane (PU) composite (d-CC@PU) and the g-CMCs with PU composite (g-CMC@PU) were obtained by dispersing d-CCs and g-CMCs in PU, respectively. The electromagnetic wave shielding properties of d-CC@PU and g-CMC@PU composites were investigated in the frequency range of 0.25 - 4.0 GHz. The shielding effectiveness (SE) of d-CC@PU and g-CMC@PU composites were measured and discussed according to the weight percent of d-CCs and g-CMCs in the composites with the thickness of the composites layers. On the whole frequency range in this work, the SE of g-CMC@PU composites was higher than those of d-CC@PU composites, irrespective of the weight percent of carbon coils in the composites and the layer thickness. Furthermore, we confirmed that the absorption mechanism, instead of the reflection mechanism, seemed to play the critical role to shield the EMI for not only the g-CMC@PU composites but also the d-CC@PU composites.
\end{abstract}

\section{Keywords}

Carbon Coils, Electromagnetic Wave Interference, Shielding Effectiveness, Absorption Mechanism

\section{Introduction}

Due to their unique geometry and the chirality, carbon coils were supposed to have unique electrical and optical properties that could be used in nanoelectronics [1]-[3]. In particular, carbon coils are understood to play the good electromagnetic wave absorbers because the coil geometry had an effective form for inducing current

${ }^{*}$ Corresponding author.

How to cite this paper: Kang, G.-H., Kim, S.-H. and Kim, S. (2015) Enhancement of the Electromagnetic Wave Shielding Effectiveness by Geometry-Controlled Carbon Coils. Journal of Materials Science and Chemical Engineering, 3, 37-44.

http://dx.doi.org/10.4236/msce.2015.31006 
through an inductive electromotive force [1] [2]. Furthermore, the demand of lightweight and moldable materials for the portable electronic devices, the polymer-matrix composites, instead of the metal-based materials, for the electromagnetic interference (EMI) shielding materials are more and more required [4] [5]. In this respect, carbon coils are regarded as the promising candidates for EMI shielding materials.

In general, the geometry of carbon coils (d-CCs) was known to be very diverse at as-grown state [6]. Furthermore, their diameter could be varied from the nano-size to the micro-size scale. So, the electrical properties of the carbon coils could be varied depending on their geometry including the diameter, as in case of carbon nanotubes [7]. For the practical application of carbon coils, therefore, it is essential to achieve the geometrically controlled carbon coils. In our previous reports, the continuous injection of $\mathrm{SF}_{6}$ gas flow during the overall reaction could give rise to d-CCs [8]. The dominant formation of the geometrically controlled carbon microcoils (g-CMCs) could be also obtained by manipulating the incorporation of $\mathrm{SF}_{6}$ flow during the reaction [9] [10]. With regard to the manipulating the incorporation of $\mathrm{SF}_{6}$ flow, our previous reports strongly confirmed that the formation of the geometrically controlled carbon coils is possible by the in situ cycling on/off modulation process employing $\mathrm{C}_{2} \mathrm{H}_{2} / \mathrm{SF}_{6}$ flow [10]. The in situ cycling on/off modulation process can be simply achieved by turning a gas flow rate on or off during the reaction. Furthermore, it was understood that the increased number of cyclic on/off modulation processes of $\mathrm{C}_{2} \mathrm{H}_{2} / \mathrm{SF}_{6}$ flow suppresses the formation of carbon microcoils (CMCs), while developing carbon nanocoils (CNCs) [9].

In this work, we investigated the shielding properties of the d-CCs and g-CMCs in the polymer composites. The d-CCs with polyurethane (PU) composite (d-CC@ PU) and g-CMCs with PU composite (g-CMC@PU) were obtained by dispersing d-CCs and g-CMCs in PU, respectively. The electromagnetic wave shielding properties of d-CC@PU or g-CMC@PU composites were measured according to the weight percent of d-CCs or g-CMCs in PU and the thickness of the composites layers in the frequency range of $0.25-4.0 \mathrm{GHz}$. Based on these results, we also discussed and compared the main shielding mechanism of d-CC@PU and g-CMC@PU composites.

\section{Experimental}

For the deposition of d-CCs and g-CMCs, a home-made thermal chemical vapor deposition system was employed. $\mathrm{C}_{2} \mathrm{H}_{2}, \mathrm{H}_{2}$ were used as source gases. The incorporated additive gas, $\mathrm{SF}_{6}$, was continuously or on/off cyclic injected into the reactor during the reaction. Figure 1 shows the detailed manipulation schemes for these gases flows according to the different processes with the samples.

The detailed reaction conditions were shown in Table 1.

For d-CC@PU and g-CMC@PU composites, d-CCs and g-CMCs were dispersed in PU solvent with the addition of dimethyl formamide (DMF) using ultrasonic system.

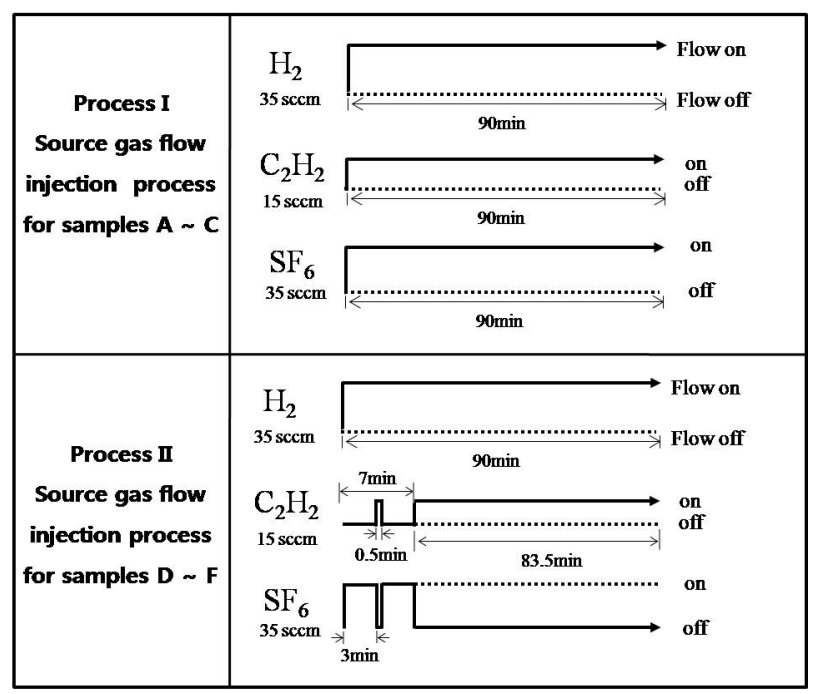

Figure 1. Schematic diagram for the manipulation schemes of $\mathrm{H}_{2}, \mathrm{C}_{2} \mathrm{H}_{2}$ and $\mathrm{SF}_{6}$ flows with the processes (the samples). 
Table 1. Experimental conditions for the deposition of carbon coils.

\begin{tabular}{|c|c|c|c|c|c|c|c|c|c|}
\hline \multirow{2}{*}{$\begin{array}{l}\text { Experimental } \\
\text { Conditions }\end{array}$} & \multirow{2}{*}{$\begin{array}{l}\mathrm{C}_{2} \mathrm{H}_{2} \\
\text { Flow } \\
\text { Rate } \\
\text { (sccm) }\end{array}$} & \multirow{2}{*}{$\begin{array}{c}\mathrm{H}_{\mathbf{2}} \\
\text { Flow } \\
\text { Rate } \\
\text { (sccm) }\end{array}$} & \multirow{2}{*}{$\begin{array}{c}\text { SF }_{6} \\
\text { Flow } \\
\text { Rate } \\
\text { (sccm) }\end{array}$} & \multirow{2}{*}{$\begin{array}{l}\text { Total } \\
\text { Pressure } \\
\text { (Tom) }\end{array}$} & \multirow{2}{*}{$\begin{array}{l}\text { Total } \\
\text { Deposition } \\
\text { Time } \\
\text { (min) }\end{array}$} & \multicolumn{3}{|c|}{$\begin{array}{c}\text { Source gases flow } \\
\text { Time (min) }\end{array}$} & \multirow{2}{*}{$\begin{array}{l}\text { Substrate } \\
\text { temperature } \\
\left({ }^{\circ} \mathrm{C}\right)\end{array}$} \\
\hline & & & & & & $\mathrm{C}_{2} \mathrm{H}_{2}$ & $\mathrm{H}_{2}$ & $\mathrm{SF}_{6}$ & \\
\hline $\begin{array}{c}\text { Samples } \\
A \sim C\end{array}$ & 15 & 35 & 35 & 100 & 90 & 90 & 90 & 90 & 750 \\
\hline $\begin{array}{l}\text { Samples } \\
\text { D } \sim \text { F }\end{array}$ & 15 & 35 & 35 & 100 & 90 & 84 & 90 & 6 & 750 \\
\hline
\end{tabular}

The paste-type d-CC@PU and g-CMC@PU samples could be obtained by 120 min on/off ultrasonic treatment at $500 \mathrm{~W}$ and $20 \mathrm{kHz}$. Six kinds of the samples (samples A - F) having the different d-CCs or g-CMCs composition ratios in the paste type mixtures were prepared as shown in Table 2. Indeed, we made two different sample groups. One group has three kinds of the samples (samples A, B, and C) having the different d-CCs composition ratios in the paste type d-CC@PU mixtures and another group has three kinds of the samples (samples D, E, and F) having the different g-CMCs composition ratios in the paste type g-CMC@PU mixtures.

After manufacturing six kinds of paste-type samples, each sample was coated onto the circular-shaped glass plate. The variation of the thickness for the coated layers of the samples was measured as a function of the number of coatings. The time intervals between the coatings were almost 24 hours. The thickness of the coated layers was estimated using the cross section images of scanning electron microscopy.

The SE of the composites was analyzed using a network analyzer (SynthNV2_3b, Windfreak Tech.). The set-up consisted of a sample holder with its outside connecting to the network analyzer. The coaxial sample holder (Electro Matrix EM-2107A) and the coaxial transmission test specimen were set according to the method of ASTM D4935-99 as shown in Figure 2. The performance measurement range of the SE for the composites was from $250 \mathrm{MHz}$ to $4.0 \mathrm{GHz}$.

The morphologies of the carbon coils-deposited on substrates were investigated in detail using field emission scanning electron microscopy (FESEM, Hitachi S-4200).

\section{Results and Discussion}

Figure 3 shows FESEM images of the surface morphologies of the substrate performed by the continuous $\mathrm{C}_{2} \mathrm{H}_{2}$ $+\mathrm{H}_{2}+\mathrm{SF}_{6}$ gas flow process (see the process I in Figure 1). Figure 3(b) shows the magnified image for Figure 3(a).

As shown in these figures, the formation of carbon nanocoils (CNCs), double helix type carbon microcoils (dh-CMCs), single helix type carbon microcoils (sh-CMCs), linear type carbon fibers (CNFs), could be observed on the whole surface of the sample. These results reveal that the continuous $\mathrm{C}_{2} \mathrm{H}_{2}+\mathrm{H}_{2}+\mathrm{SF}_{6}$ gas flow process can give rise to the formation of d-CCs.

Figure 4 shows FESEM images of the surface morphologies of the substrate performed by the cyclic modulation process of $\mathrm{C}_{2} \mathrm{H}_{2}$ and $\mathrm{SF}_{6}$ gas (see process II in Figure 1). Figure 4(b) shows the magnified image for Figure 4(b). As shown in these figures, the well-structured CMCs were prevailed on the surface of the sample. These results reveal that the process II can give rise to the dominant formation of the CMCs.

As shown in Figure 5(a) and Figure 5(b), the thicknesses of the coated layers were gradually increased with the number of coatings. Although we poured almost same amount ( $20 \mathrm{ml})$ of the paste-type sample for every coating, the increased thickness of the coated layer of the sample was not directly proportional to the number of coatings. It seemed to be due to the volatile DMF which had been evaporated during the natural drying process. As expectation, the highest composition ratios of d-CCs in d-CC@PU composites (sample C) and the highest composite ratios of g-CMCs in g-CMC@PU composites (sample F) gave rise to the highest thickness of the coated layers among the samples A - C, and the samples D - F, respectively.

After measuring the thickness of the coated layer of the sample, we examined the variation tendency of SE according to the different composition ratios of carbon coils in the composites and the different thickness of the composites as shown in Figures 6-8.

On the whole frequency range in this work, the SE of g-CMC@PU composites was higher than those of d-CC@PU composites, irrespective of the weight percent of carbon coils in the composites and the layer thickness. These results confirmed that the g-CMC@PU composite is more effective to work as a shielding material of EMI. 

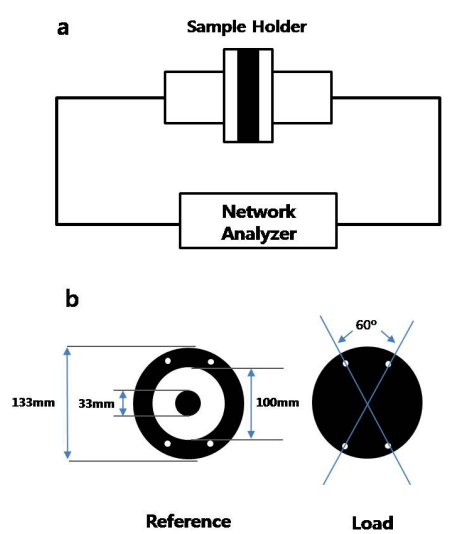

Figure 2. (a) Instrumental setup measuring the shielding effectiveness; (b) Coaxial transmission test specimen according to the method of ASTM D4935-99.

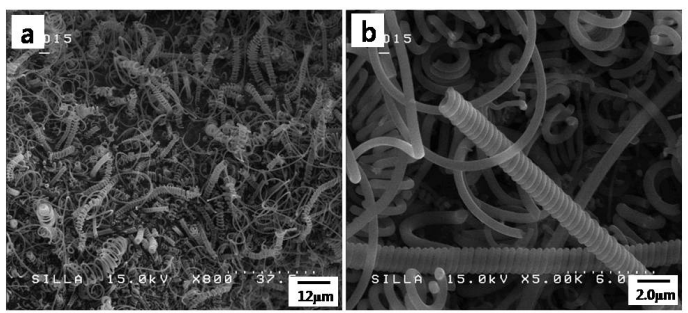

Figure 3. (a) The representative FESEM images of the surface morphologies of the substrate performed by the process II and (b) the magnified image of Figure 3(a).
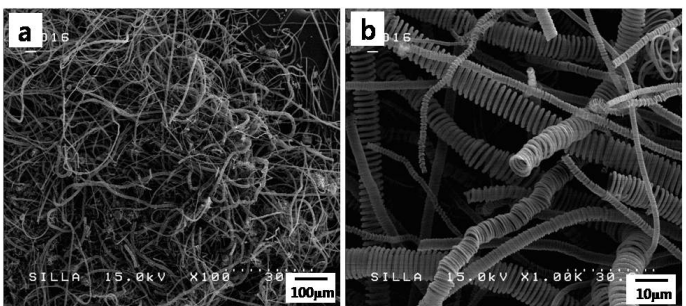

Figure 4. (a) The representative FESEM images of the surface morphologies of the substrate performed by the process II and (b) the magnified image of Figure 4(a).

Table 2. Six kinds of the samples (samples A - F) having the different d-CCs, g-CMCs, PU, and DMF composition ratios in the paste type mixtures.

\begin{tabular}{llcccc}
\hline & & \multicolumn{4}{c}{ Weight \% } \\
\cline { 3 - 6 } Groups & Samples & d-CCs & g-CMCs & PU & DMF \\
\hline \multirow{2}{*}{ Group I } & Sample A & $\sim 5$ & - & $\sim 67$ & $\sim 27$ \\
& Sample B & -10 & - & $\sim 64$ & -26 \\
& Sample C & $\sim 16$ & - & $\sim 67$ & $\sim 17$ \\
\hline \multirow{2}{*}{ Group II } & Sample D & - & $\sim 4$ & $\sim 67$ & $\sim 29$ \\
& Sample E & - & -11 & $\sim 69$ & -20 \\
& Sample F & - & $\sim 17$ & $\sim 66$ & $\sim 17$ \\
\hline
\end{tabular}



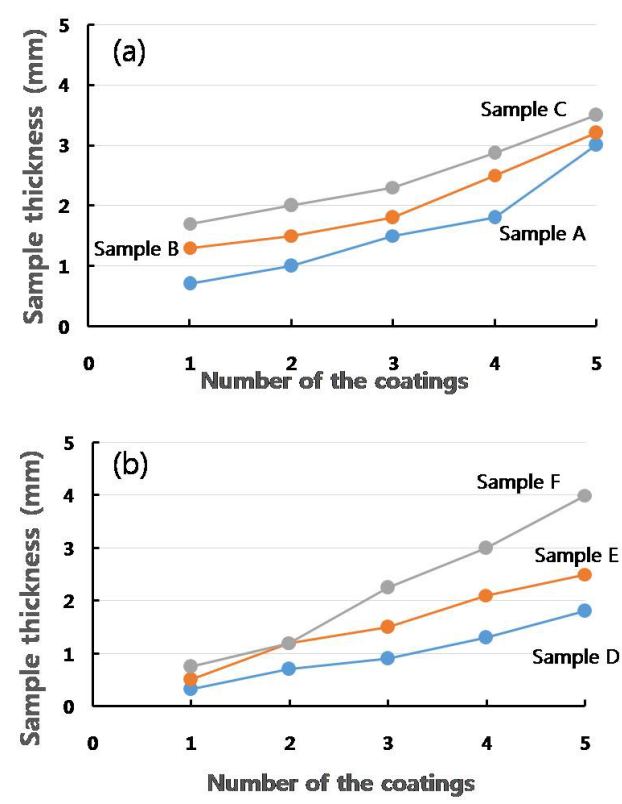

Figure 5. Variation of the coated-layer thickness for different samples as a function of the number of coatings.

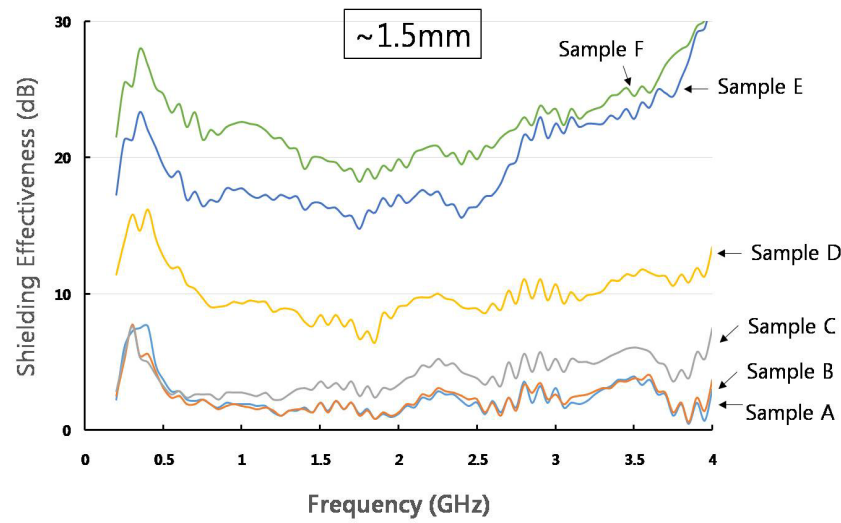

Figure 6. The variation of the shielding effectiveness according to the samples at $\sim 1.5 \mathrm{~mm}$ composite layer thickness.

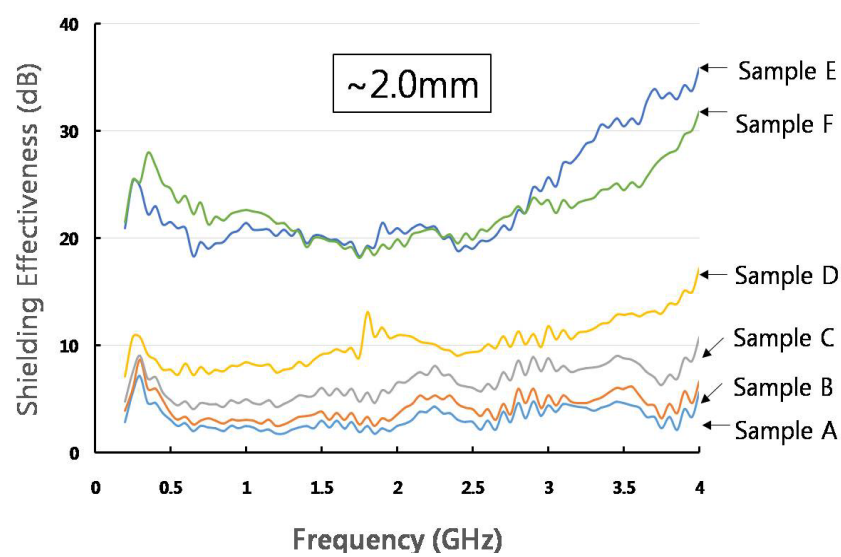

Figure 7. The variation of the shielding effectiveness according to the samples at $\sim 2.0 \mathrm{~mm}$ composite layer thickness. 


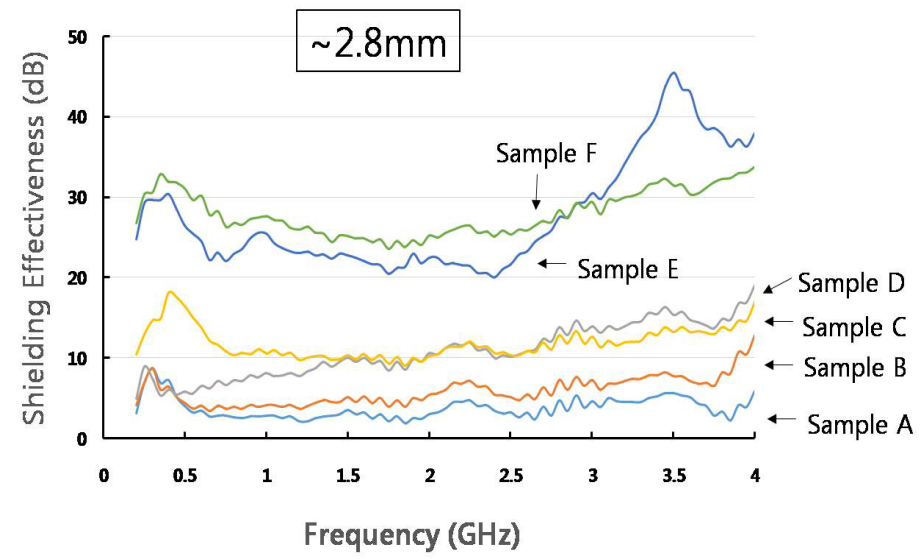

Figure 8. The variation of the shielding effectiveness according to the samples at $\sim 2.8 \mathrm{~mm}$ composite layer thickness.

Meanwhile, reflection, absorption, and multiple reflections are thought as the major three mechanisms to shield EMI [11]. In the conducting materials like metals, the shielding is usually enhanced by the electrical conductivity of the materials [11] [12]. So, reflection is understood to work as the main shielding mechanism to protect EMI [12] [13]. For the materials having the high electric constants or the magnetic permeability, absorption was known as the major shielding mechanism [11]. For the multiple reflections, the small-sized fillers having the high surface area in the composite usually gave rise to the better shielding performance [11]. These three mechanisms can complementary and/or supplementary operate to shield the EMI.

Considering only the reflection and the absorption effects as the main shielding mechanisms for EMI of this work, the SE of EMI for the electrically conductive polymer composites can be estimated by the empirical equation of Simon [14]:

$$
S E=50+10 \log _{10}(r f)^{-1}+1.7 t(f / r)^{1 / 2}
$$

where $\mathrm{SE}$ is in $\mathrm{dB}, r$ is the volume resistivity (Wcm) at room temperature, $t$ is the thickness of the sample (cm), $f$ is the measurement frequency, respectively. In this equation, the combined first and second terms, namely $50+$

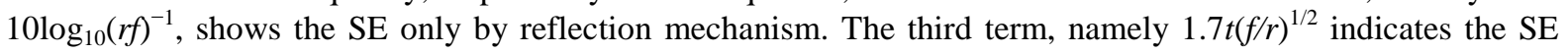
only by absorption mechanism.

We first compared the SE of d-CC@PU composites for samples A - C and the SE of g-CMC@PU composites for samples D - F under the similar composite layer thickness as shown in Figures 6-8. The samples C, F seems to have the highest $\mathrm{SE}$ among the respective sample group. It indicates that the reflection effect may work as the SE mechanism for the EMI in this case, because only the d-CCs and g-CMCs can have the electrical conductive characteristics in d-CC@PU composite and in g-CMC@PU composites. As indicated by Simon's equation, the SE from reflection decreases with increasing the measurement frequency, while the SE from absorption increases with increasing the measurement frequency. For the measuring frequency dependence of the SE for the samples, the samples show the increase of SE with increasing the measurement frequency in the range of $0.25-4.0$ GHz. These results inform that the absorption may work as the main SE mechanism for the EMI of these composites.

For the composite layer thickness dependence, the third term of Simon's equation, $\left.1.7 t(f / r)^{1 / 2}\right)$, indicates that the SE by absorption mechanism increases with increasing the thickness $(t)$. Figure 9 and Figure 10 show the variation of the $\mathrm{SE}$ as a function of the measurement frequency for samples $\mathrm{C}$ and $\mathrm{F}$, respectively. These figures clearly indicate that the SE of the sample increases with increasing the coated layer thickness. Therefore, we confirm that the absorption would play as the main shielding mechanism to protect EMI for in the d-CC@PU composites as well as in the g-CMC@PU composites.

\section{Conclusion}

On the whole frequency range in this work, the g-CMC@PU composite is more effective to work as a shielding 


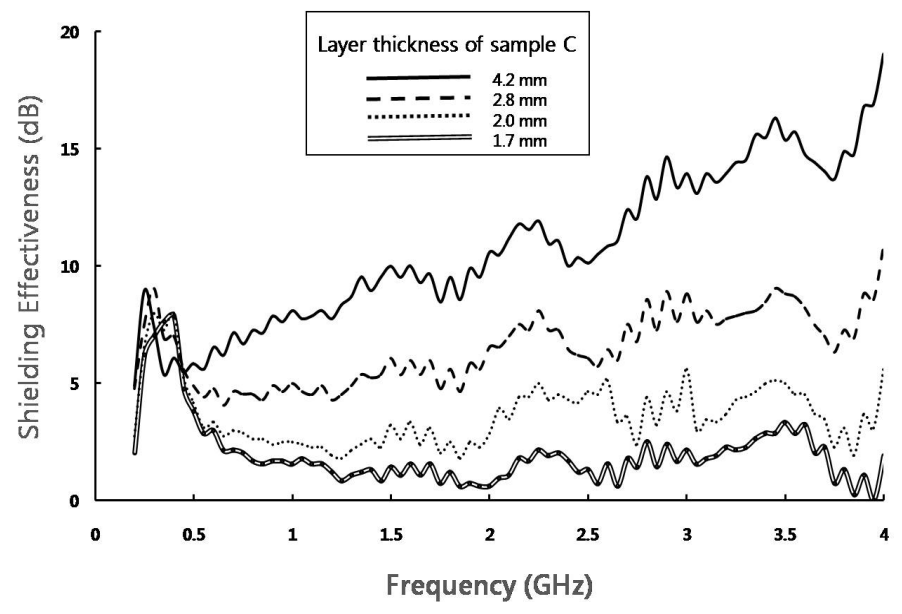

Figure 9. The variation of the shielding effectiveness of sample $\mathrm{C}$ according to the different thickness of the coated layers.

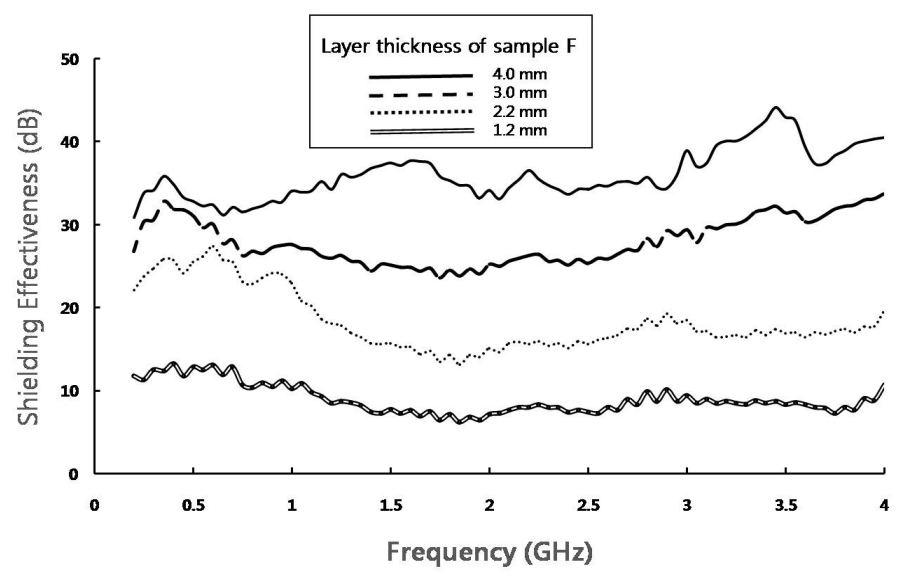

Figure 9. The variation of the shielding effectiveness of sample F according to the different thickness of the coated layers.

material of EMI. The absorption is the main mechanism to shield the EMI for d-CC@PU composite as well as g-CMC@PU composite.

\section{References}

[1] Amelinckx, S., Zhang, X.B., Bernaerts, D., Zhang, X.F., Ivanov, V. and Nagy, J.B. (1996) A Formation Mechanism for Catalytically Grown Helix-Shaped Graphite Nanotubes. Science, 265, 635-639.

[2] Zhao, D.-L. and Shen, Z.-M. (2008) Preparation and Microwave Absorption Properties of Carbon Nanocoils. Materials Letters, 62, 3704-3706. http://dx.doi.org/10.1016/j.matlet.2008.04.032

[3] Motojima, S., Hoshiya, S. and Hishikawa, Y. (2003) Electromagnetic Wave Absorption Properties of Carbon Microcoils/PMMA Composite Beads in W Bands. Carbon, 41, 2658-2660. http://dx.doi.org/10.1016/S0008-6223(03)00292-6

[4] Yang, L., Gupta, M.C., Dudley, K.L. and Lawrence, R.W. (2005) Novel Carbon Nanotube-Polystyrene Foam Composites for Electromagnetic Interference Shielding. Nano Letters, 5, 2131-2135. http://dx.doi.org/10.1021/nl051375r

[5] Wu, J. and Chung, D.D.L. (2003) Improving Colloidal Graphite for Electromagnetic Interference Shielding Using 0.1 $\mu \mathrm{m}$ Diameter Carbon Filaments. Carbon, 41, 1313-1315. http://dx.doi.org/10.1016/S0008-6223(03)00033-2

[6] Shaikjee, A. and Coville, N.J. (2012) The Synthesis, Properties and Uses of Carbon Materials with Helical Morphology. Journal of Advanced Research, 3, 195-223. http://dx.doi.org/10.1016/j.jare.2011.05.007 
[7] Akagi, K., Tamura, R., Tsukada, M., Itoh, S. and Ihara, S. (1995) Electronic Structure of Helically Coiled Cage of Graphitic Carbon. Physical Review Letters, 74, 2307-2310. http://dx.doi.org/10.1103/PhysRevLett.74.2307

[8] Eum, J.-H., Kim, S.-H., Yi, S.S. and Jang, K. (2012) Large-Scale Synthesis of the Controlled-Geometry Carbon Coils by the Manipulation of the $\mathrm{SF}_{6}$ Gas Flow Injection Time. Journal of Nanoscience and Nanotechnology, 12, 4397-4402. http://dx.doi.org/10.1166/jnn.2012.5940

[9] Eum, J.-H., Jeon, Y.-C. and Kim, S.-H. (2012) Effect of Gas Phase Composition Cycling on/off Modulation Numbers of $\mathrm{C}_{2} \mathrm{H}_{2} / \mathrm{SF}_{6}$ Flows on the Formation of Geometrically Controlled Carbon Coil. Journal of Nanoscience and Nanotechnology, 12, 6100-6106. http://dx.doi.org/10.1166/jnn.2012.6342

[10] Jeon, Y.-C., Eum, J.-H., Kim, S.-H., Park, J.-C. and Ahn, S.I. (2012) Effect of the on/off Cycling Modulation Time Ratio of $\mathrm{C}_{2} \mathrm{H}_{2} / \mathrm{SF}_{6}$ Flows on the Formation of Geometrically Controlled Carbon Coils. Journal of Nanomaterials, 2012, Article ID: 908961.

[11] Wu, J. and Chung, D.D.L. (2001) Increasing the Electromagnetic Interference Shielding Effectiveness of Carbon Fiber Polymer-Matrix Composite by Using Activated Carbon Fibers. Carbon, 40, 445-467. http://dx.doi.org/10.1016/S0008-6223(01)00133-6

[12] Sau, K.P., Chaki, T.K., Chakraborty, A. and Khastgir, D. (1997) Electromagnetic Interference Shielding by Carbon Black and Carbon Fiber Filled Rubber Composite. Plastics Rubber Comp. Process. Appl., 26, 291-297.

[13] Yang, S., Lozano, K., Lomeli, A., Foltz, H.D. and Jones, R. (2005) Electromagnetic Interference Shielding Effectiveness of Carbon Nanofiber/LCP Composites. Composites: Part A, 36, 691-697. http://dx.doi.org/10.1016/j.compositesa.2004.07.009

[14] Simon, R.M. (1981) EMI Shielding through Conductive Plastics. Polymer-Plastics Technology and Engineering, 17, 1-10. http://dx.doi.org/10.1080/03602558108067695 